\title{
Predisposing Factors for Intraoperative Endplate Injury of Extreme Lateral Interbody Fusion
}

\author{
Kotaro Satake, Tokumi Kanemura, Hidetoshi Yamaguchi, Naoki Segi, Jun Ouchida \\ Department of Orthopaedic Surgery, Konan Kosei Hospital, Konan, Japan
}

\section{Study Design: Retrospective study.}

Purpose: To compare intraoperative endplate injury cases and no injury cases in consecutive series and to identify predisposing factors for intraoperative endplate injury.

Overview of Literature: Unintended endplate violation and subsequent cage subsidence is an intraoperative complication of extreme lateral interbody fusion (XLIF). It is still unknown whether it is derived from inexperienced surgical technique or patients' inherent problems.

Methods: Consecutive patients ( $\mathrm{n}=102$; mean age, $69.0 \pm 0.8$ years) underwent XLIF at 201 levels at a single institute. Preoperative and immediately postoperative radiographs were compared and cases with intraoperative endplate injury were identified. Various parameters were reviewed in each patient and compared between the injury and no injury groups.

Results: Twenty one levels (10.4\%) had signs of intraoperative endplate injury. The injury group had a significantly higher rate of females $(p=0.002)$, lower bone mineral density (BMD) $(p=0.02)$, higher rate of polyetheretherketone as cage material $(p=0.04)$, and taller cage height $(p=0.03)$ compared with the no injury group. Multivariate analysis indicated that a T-score of BMD as a negative (odds ratio, $0.52 ; 95 \%$ confidence interval, $0.27-0.93 ; p=0.03$ ) and cage height as a positive (odds ratio, $1.84 ; 95 \%$ confidence interval, 1.01-3.17; $p=0.03$ ) were predisposing factors for intraoperative endplate injury.

Conclusions: Intraoperative endplate injury is correlated significantly with reduced BMD and taller cage height. Precise evaluation of bone quality and treatment for osteoporosis might be important and care should be taken not to choose excessively taller cage.

Keywords: Bone-implant interface; Minimally invasive surgical procedure; Osteoporosis; Spine; Complication

\section{Introduction}

In the extreme lateral interbody fusion (XLIF) procedure, restoring disc height is one of the important goals in achieving indirect decompression of neural elements and restoration of segmental lordosis at the surgical site. However, these distraction effects are sometimes compromised by endplate injury and subsequent cage subsidence. Thus, endplate injury is considered to be a serious intraoperative complication in the XLIF procedure [1-3].

Several reports have described postoperative cage subsidence in the XLIF procedure, with an incidence ranging from $0.3 \%$ to $22 \%$ [1-3]. However, almost all reports did not separately analyze the two types of cage subsidence; one occurs gradually in the postoperative course (spontaneous type) and the other is derived from intraoperative

Received Jan 26, 2016; Revised Feb 29, 2016; Accepted Mar 28, 2016

Corresponding author: Kotaro Satake

Department of Orthopaedic Surgery, Konan Kosei Hospital,

137 Takayamachi Omatsubara, Konan 483-8704, Japan

Tel: +81-587-51-3333, Fax: +81-587-51-3300, E-mail: k-satake@konan.jaaikosei.or.jp 
endplate injury (iatrogenic type). Tohmeh et al. [4] first analyzed these two types separately and compared their radiographic and clinical outcomes in a XLIF series. The spontaneous type was a normal process of endplate remodeling caused by biomechanical loading, while the iatrogenic type was an intraoperative complication that could cause progressive cage subsidence into the vertebral body and end up in failure of indirect decompression and bony fusion. The authors reported that more than half of the cases of intraoperative endplate injury demonstrated progressive settling with a larger magnitude and lower clinical improvement than the cases without injury. A biomechanical study supported these clinical results by demonstrating that endplate removal significantly decreased the failure load [5].

Special care is recommended for endplate preparation and cage insertion during XLIF procedure [1]. Unintended endplate injury has been attributed only to poor surgical technique [4] and it is not yet clear whether it is derived only from surgical skill or whether it is related to a patient's inherent condition. The present study focused on this intraoperative endplate injury with the aim of identifying its predisposing factors in our initial consecutive XLIF series.

\section{Materials and Methods}

\section{Patient population}

A retrospective review study was performed with Institutional Review Board approval. The study included consecutive patients who underwent XLIF (NuVasive Inc., San Diego, CA, USA) at the institute of the authors (Konan Kosei Hospital) from February 2013 to February 2015. The 102 enrolled patients comprised 41 males and $61 \mathrm{fe}$ males, with a mean age at surgery of $69.0 \pm 0.8$ years, and a total of 201 levels. Preoperative diagnosis was degenerative scoliosis/kyphoscoliosis in 38 patients, spondylolisthesis in 29 , adjacent segmental disease in 12 , thoracic or lumbar canal stenosis in 13, congenital or syndromic scoliosis in 5, and other diagnoses in 5. Patient demographics are presented in Table 1.

\section{Surgical details}

Operations were performed by 6 surgeons, however all cases were under supervision of one of the senior authors
Table 1. Patient's demographics

\begin{tabular}{lc}
\hline Characteristic & Patients (n=102) \\
\hline Age (yr) & $69.0 \pm 0.8$ \\
\hline Sex: female & $61(60.6)$ \\
\hline Previous vertebral fracture: yes & $18(17.6)$ \\
\hline BMD (T-score) & $-1.36 \pm 0.13$ \\
\hline Diagnosis & \\
\hline Degenerative scoliosis/kyphoscoliosis & $38(37.3)$ \\
\hline Spodylolisthesis & $29(28.4)$ \\
\hline Adjacent segmental disease & $12(12.1)$ \\
\hline Stenosis & $13(12.7)$ \\
\hline Congenital/syndromic scoliosis & $5(4.9)$ \\
\hline Others & $5(4.9)$ \\
\hline Total number of levels & $201(2.01$ per patient) \\
\hline Surgical site & \\
\hline Thoracic spine & $17(8.5)$ \\
\hline Upper lumbar spine (L1-L2, L2-L3) & $52(25.9)$ \\
\hline Lower lumber spine (L3-L4, L4-L5) & $132(65.7)$ \\
\hline
\end{tabular}

Values are presented as mean \pm SE or number (\%).

$n$, number of patients or levels; BMD, bone mineral density; SE, standard error.

(T. K.). Procedures strictly adhered to the surgical technique described by Ozgur et al. [6]. For endplate preparation, a surface marking with soft indentation made by a box cutter was followed by annulus incision with a knife. After the removal of the disc material by a rongeur, a Cobb elevator was advanced gently along the endplates to release the contralateral annulus under fluoroscopy guidance. Cage size trials were followed by additional disc curettage and rasping of the endplates. A box cutter was not used routinely for disc removal, only for younger patients with larger disc height of more than $11 \mathrm{~mm}$. The sizing of the cage was based on the touch and feel of the trial cage inserted into the disc space. All cages were inserted using two containment sliders to protect the endplates and to keep the graft material inside the cage. For the initial 16 patients (32 levels), titanium cages of standard $18 \mathrm{~mm}$ width (CoRoent XL, NuVasive) were used. Later, polyetheretherketone (PEEK) cages of the same width were used for all residual patients. As for graft material, the last 8 patients (18 levels) received artificial bone material composed of hydroxyapatite and collagen (Refit, HOYA Technosurgical, Tokyo, Japan) soaked in auto-bone marrow aspirate. The others received allograft bone. All patients 
were supplemented with bilateral pedicle screw fixation.

\section{Data collection}

Patient demographic and surgical details were reviewed from the clinical charts. Age at surgery, sex, history of previous vertebral fractures, $\mathrm{T}$-score for bone mineral density (BMD) measured at the left femoral neck using dual-energy X-ray absorptiometry (DEXA) to avoid the influence of the previously instrumented metals in lumbar spine of some cases, surgeon experience, surgical level (thoracic, upper lumbar [L1/L2 and L2/L3], and lower lumbar [L3/ L4 and L4/L5]), the material (titanium or PEEK), height (8-14 mm), length $(40-60 \mathrm{~mm})$, and lordosis $\left(0^{\circ}\right.$ or $\left.10^{\circ}\right)$ of the cages were investigated (Table 1 ). Concerning surgeon experience, 3 surgeons were classified as low volume (experienced 1-10 levels), 2 were middle volume (experienced 11-30 levels), and one was high volume (experienced $\geq 31$ levels).

\section{Radiographic measurements}

Radiographic assessment was independently performed by two spinal surgeons (H.Y. and N.S.) blinded to the study information. A third reviewer (T.K.) was available for adjudication in case of disagreement. Preoperative lateral X-ray in the standing position and immediate postoperative X-ray taken in the prone position on the operating table were compared at each surgical level, and every cage subsidence more than $2 \mathrm{~mm}$ combined with endplate injury was identified (Fig. 1). They were classified as the injury group. Surgical levels with no endplate injury were classified as the no injury group.

Radiographic measurements were performed as follows.
From the preoperative computed tomography multiplanar reconstruction (CT-MPR), each disc height at the endplate's center of the anterior-posterior border of the vertebra (DH) and segmental lordosis (angulation between the inferior and superior endplates facing each other [SL]) were measured in the sagittal midline slice (Fig. 2A). In deformity patients, a slice showing both rostral and caudal spinal processes was chosen for these measurements. Based on these measurements, the disc height gap in $\mathrm{mm}$ was calculated as cage height-preoperative disc height. The segmental lordosis gap in degrees was also calculated as cage lordosis-preoperative segmental lordosis. These calculations estimated how much the surgeon intended to distract the segment. From the preoperative coronal plane of CT-MPR, coronal segmental angle (angulation between the inferior and superior endplates facing each other, closing angle to the approach side determined as positive value; CSA) (Fig. 2B) was measured. The closest slice to the posterior one-third of the disc was chosen for this measurement. To assess the magnitude of lumbar curve, T12L4 Cobb angle was measured in a global anteriorposterior $\mathrm{X}$-ray taken in the standing position.

From postoperative CT-MPR, the position of the cage in the sagittal view was quantified. At the median slice of the sagittal plane, the center (midpoint of anterior and posterior cage edge) of the cage and the disc (midpoint of anterior and posterior vertebral edge) were identified. The distance between these two points was measured in $\mathrm{mm}$ (positive value for anterior deviation of the cage from the disc center) (Fig. 3).

\section{Statistical analyses}

Each parameter of the patient's demographic, surgical
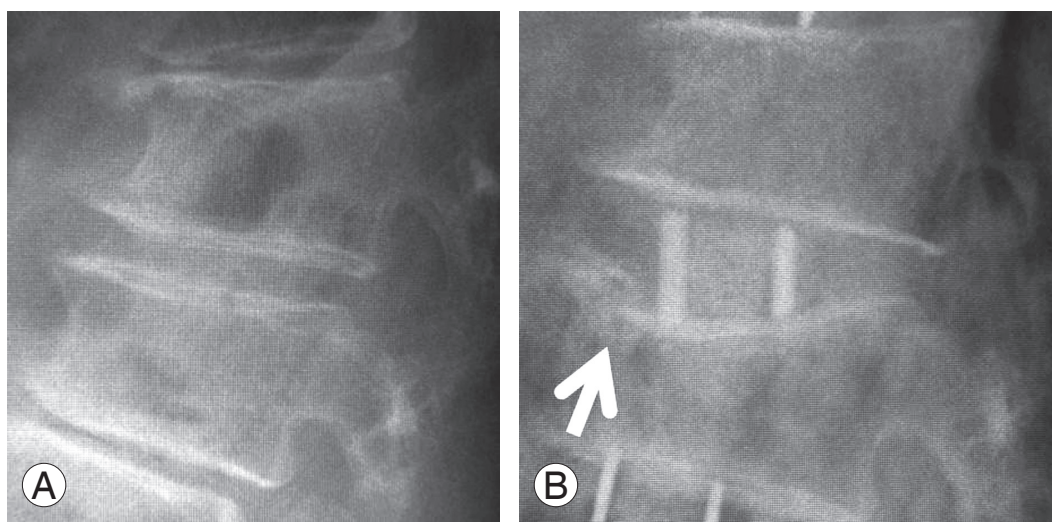

Fig. 1. Preoperative and postoperative $\mathrm{X}$-ray images. (A) Preoperative lateral $\mathrm{X}$-ray image. (B) Immediate postoperative lateral $\mathrm{X}$-ray taken at the same level as $A$. White arrow indicates an endplate injury at the superior endplate of the caudal vertebra. This level was classified as the injury group. 

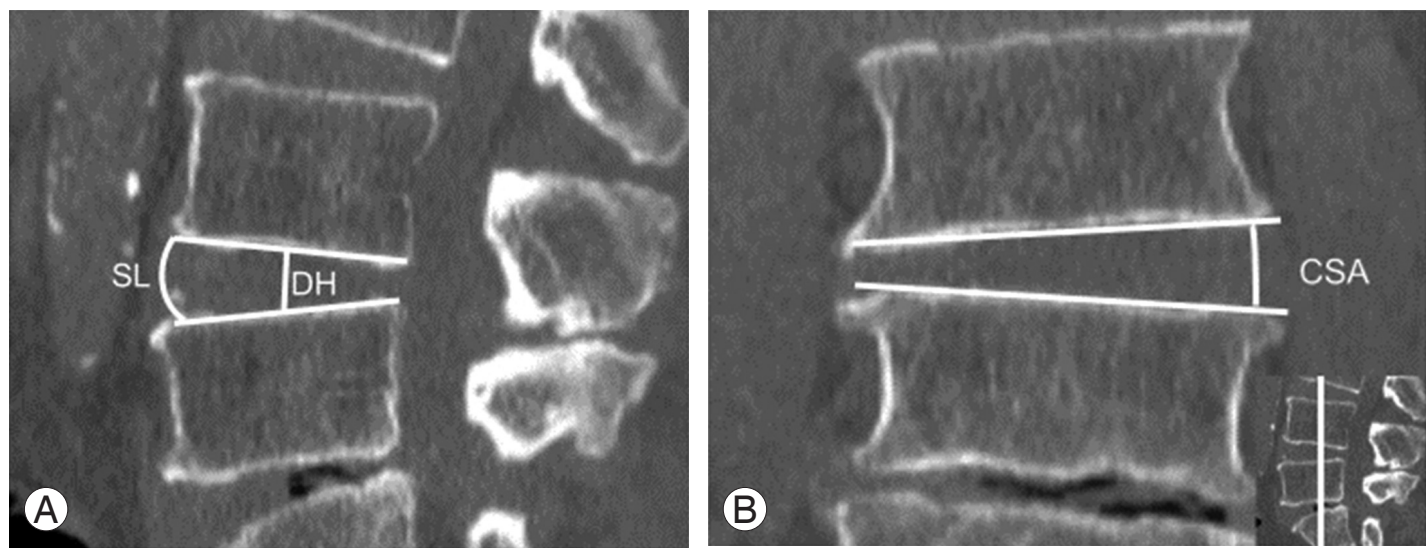

Fig. 2. Preoperative image slice data. (A) Preoperative sagittal slice of computed tomography multiplanar reconstruction (CTMPR) showing the measurements of disc height (DH) and segmental lordosis (SL). (B) Preoperative coronal slice of CT-MPR at the posterior one-third of the segment (a coronal slice at the white line in the picture at the lower right corner) showing the measurement of coronal segmental angle (CSA). The angle closing to the approach side is determined as positive value.

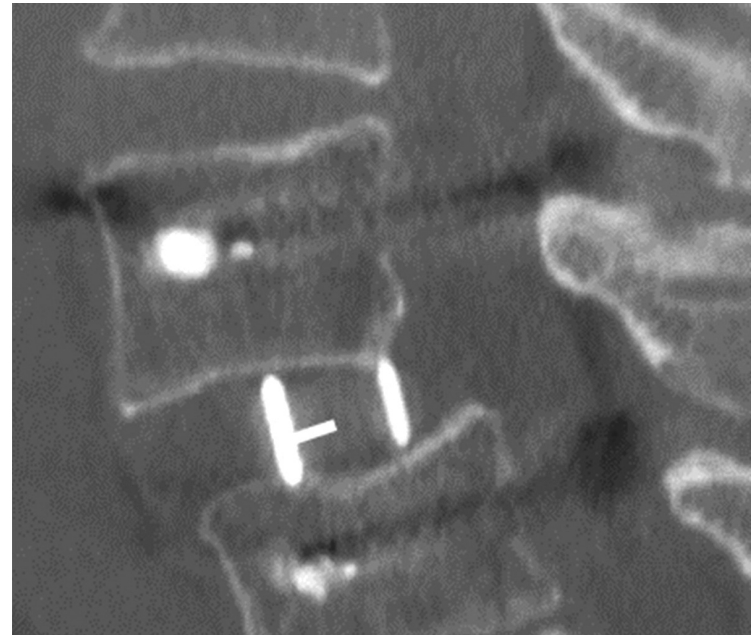

Fig. 3. Sagittal slice showing the measurement of cage position (a white line showing the deviation of the cage center from the disc center).

details, and radiographic measurements was compared between the injury and no injury groups using univariate analyses. Unpaired Student's $t$ test was used for continuous variables between two groups. If the distribution of variables was not equal between the two groups, MannWhitney U test was used. Chi-square test or Fisher's exact test was used for dichotomous and categorical variables. A $p$-value $<0.05$ was accepted as significant. Multivariate logistic regression was then performed to determine the predisposing factor for the intraoperative endplate injury. All univariate variables that were significantly different between the two groups were entered into a backward stepwise multiple logistic regression analysis. All analyses

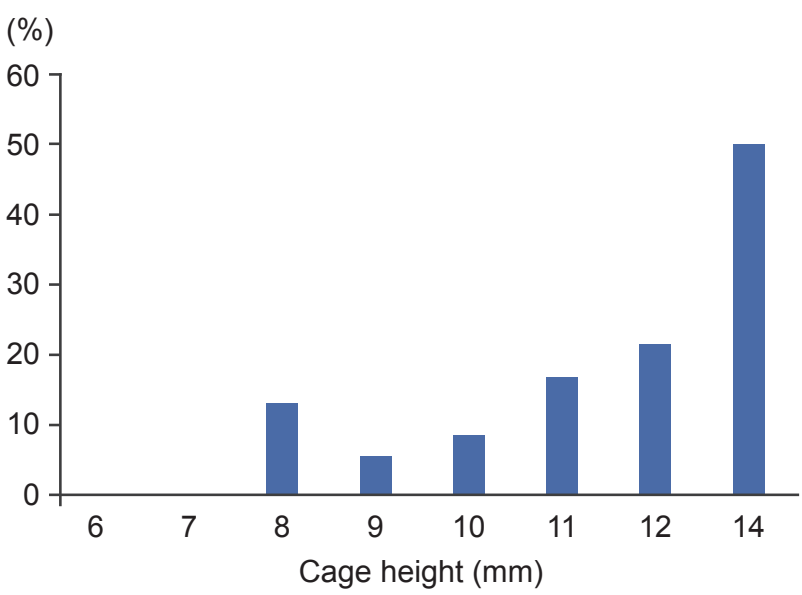

Fig. 4. Incidence of endplate injury of each cage height.

were performed using IBM SPSS Statistics ver. 19 (IBM. Armonk, NY, USA).

\section{Results}

In 21 levels (10.4\%) of 17 patients, postoperative cage subsidence subsequent to intraoperative endplate injury was identified. Nineteen injury cases were observed at the superior endplate, one at the inferior, and one at both. The injury group consisted of 20 levels of females and only one level of male. This difference of patient's sex was statistically significant $(p=0.002)$. T-score of BMD was significantly lower in the injury group $(p=0.02)$. Cage height was significantly higher in the injury group than the no injury group $(p=0.03)$. The incidence of endplate injury increased linearly as cage height increased (Fig. 4). 
Table 2. Univariate analyses between the injury- and the no injury group

\begin{tabular}{|c|c|c|c|}
\hline Characteristic & $\begin{array}{l}\text { Injury group } \\
(n=21,10.4 \%)\end{array}$ & $\begin{array}{c}\text { No injury group } \\
(n=180,89.6 \%)\end{array}$ & $p$-value \\
\hline Age (yr) & $71.6 \pm 2.0$ & $68.7 \pm 0.9$ & 0.26 \\
\hline Sex: female & $20(95.2)$ & $111(61.7)$ & 0.002 \\
\hline Previous vertebral fracture: yes & $6(28.6)$ & $37(20.6)$ & 0.4 \\
\hline BMD (T-score) & $-2.14 \pm 0.16$ & $-1.24 \pm 0.15$ & 0.02 \\
\hline Diagnosis & & & 0.25 \\
\hline Degenerative scoliosis/kyphoscoliosis & $9(42.9)$ & $96(53.3)$ & \\
\hline Congenital/syndromic scoliosis & $2(9.5)$ & $14(7.8)$ & \\
\hline Spondylolisthesis & $7(33.3)$ & $31(17.2)$ & \\
\hline Adjacent segmental disease & 0 & $19(10.6)$ & \\
\hline Stenosis & $3(14.3)$ & $15(8.3)$ & \\
\hline Others & 0 & $5(2.8)$ & \\
\hline \multicolumn{4}{|l|}{ Preoperative $\mathrm{X}$-ray } \\
\hline Disc height (mm) & $6.3 \pm 0.6$ & $5.5 \pm 0.2$ & 0.14 \\
\hline Segmental lordosis $\left({ }^{\circ}\right)$ & $4.9 \pm 0.9$ & $4.8 \pm 0.3$ & 0.9 \\
\hline Coronal segmental angle $\left({ }^{\circ}\right)$ & $-0.1 \pm 0.9$ & $0.1 \pm 0.4$ & 0.82 \\
\hline $\mathrm{T} 12-\mathrm{L} 4 \operatorname{Cobb}\left({ }^{\circ}\right)$ & $14.6 \pm 2.5$ & $12.8 \pm 0.8$ & 0.46 \\
\hline \multicolumn{4}{|l|}{ Surgical details } \\
\hline Surgeon's experience volume & & & 0.48 \\
\hline High volume $(n=1)$ & $10(47.6)$ & $79(43.9)$ & \\
\hline Middle volume ( $n=2)$ & $4(19.0)$ & $56(31.1)$ & \\
\hline Low volume $(n=3)$ & $7(33.3)$ & $45(25.0)$ & \\
\hline Cage material & & & 0.03 \\
\hline Titanium & 0 & $32(17.8)$ & \\
\hline PEEK & $21(100)$ & $148(82.2)$ & \\
\hline Cage height $(\mathrm{mm})$ & $10.3 \pm 0.3$ & $9.7 \pm 0.1$ & 0.04 \\
\hline Cage length (mm) & $48.8 \pm 1.8$ & $49.3 \pm 0.4$ & 0.61 \\
\hline Cage lordosis $\left(0^{\circ}\right.$ or $\left.10^{\circ}\right)$ & $\begin{array}{c}10^{\circ}: 20(96.4) \\
0^{\circ}: 1(3.6)\end{array}$ & $\begin{array}{r}10^{\circ}: 153(88.4) \\
0^{\circ}: 27(11.6)\end{array}$ & 0.31 \\
\hline Fused level & $2.8 \pm 0.3$ & $2.5 \pm 0.8$ & 0.23 \\
\hline Surgical site & & & 0.5 \\
\hline Thoracic & $3(14.3)$ & $14(7.8)$ & \\
\hline Upper lumbar & $4(19.0)$ & $48(26.7)$ & \\
\hline Lower lumbar & $14(66.7)$ & $118(65.6)$ & \\
\hline Disc height gap (mm) & $4.0 \pm 0.4$ & $4.2 \pm 0.2$ & 0.73 \\
\hline Segmental lordosis gap $\left({ }^{\circ}\right)$ & $4.6 \pm 0.8$ & $3.7 \pm 0.4$ & 0.44 \\
\hline Cage position (mm) & $0.9 \pm 0.7$ & $1.6 \pm 0.2$ & 0.34 \\
\hline
\end{tabular}

Values are presented as mean \pm SE or number (\%).

n, number of patients or levels; BMD, bone mineral density; PEEK, polyetheretherketone; SE, standard error.

The incidence of endplate injury was $8.3 \%$ and $21.4 \%$ at a height of $10 \mathrm{~mm}$ and $12 \mathrm{~mm}$, respectively. Although an
$8 \mathrm{~mm}$ high cage showed higher rate $(3 / 23,13 \%)$ than a $9 \mathrm{~mm}$ or $10 \mathrm{~mm}$ cage, all three injuries were observed at 
the thoracic spine. The disc height gap and the segmental lordosis gap did not demonstrate any significant difference between the two groups. As for cage material, PEEK demonstrated significantly higher incidence of endplate injury compared with titanium $(p=0.04)$. Patient's background or radiographic parameters did not demonstrate any significant difference between the two groups (Table 2). Using multivariate logistic regression revealed T-score of $\mathrm{BMD}$ as a negative (odds ratio, $0.52 ; 95 \%$ confidence interval, $0.27-0.93 ; p=0.03$ ) and cage height as a positive (odds ratio, 1.84; 95\% confidence interval, 1.07-3.17; $p=0.03$ ) significant predisposing factor for intraoperative endplate injury (Table 3 ).

\section{Discussion}

Factors reported as causes of postoperative cage subsidence in various intervertebral fusions including XLIF are reduced bone quality $[7,8]$, older age [8] multilevel procedures $[1,9]$, narrow cage $[1,2]$, and use of recombinant human bone morphogenetic protein-2 [10]. However, these reports did not consider spontaneous and iatrogenic subsidence separately, and no report has focused on intraoperative endplate injury.

In our study, one significant parameter was lower Tscore of BMD. This means that reduced bone quality or osteoporosis significantly facilitates intraoperative endplate injury. The finding that almost all patients who suffered endplate injury were females supports the significant influence of BMD on endplate injury in this mainly postmenopausal patient population.

The endplate is cortical bone covering the superior and inferior surface of a vertebral body. The peripheral region, termed the apophyseal ring, is thicker than the central region $[8,11]$. Hou and Luo [8] analyzed biomechanical properties of lumbar endplate. The authors reported a lower failure load of endplate in vertebrae with lower BMD and concluded that patients with osteoporosis have a higher risk of cage subsidence. Grant et al. [12] demonstrated the mechanical weakness of the central region of the endplate by indentation tests. In addition, they proved that the superior endplate is mechanically weaker than the inferior endplate. This biomechanical data supported our results that show endplate injuries were dominantly observed at superior endplates. Based on biomechanical tests, Steffen et al. [13] recommended preserving the peripheral region for endplate preparation and placing the implant on the apophyseal ring. In terms of cage subsidence, XLIF has a greater advantage compared with other intervertebral fusions because of the larger footprint cage, which is able to rest on the apophyseal ring. However, it still cannot overcome the influence of osteoporosis on the endplates.

Another possible factor in intraoperative endplate injury might be poor fluoroscopic imaging of osteoporotic patients. A textbook described several technical tips to avoid intraoperative endplate injury; these included orthogonal positioning or gentle discectomy using only ring curettes [14]. In some osteoporotic cases, especially combined with severe vertebral deformity, it might be difficult to precisely

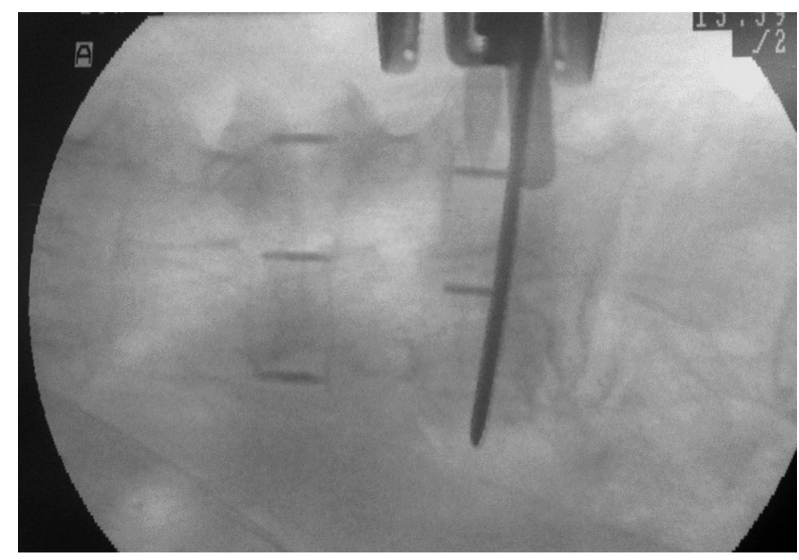

Fig. 5. Intraoperative fluoroscopic anteriorposteior image of an osteoporotic patient during cage insertion. It is difficult to identify the endplates precisely in this case.

Table 3. Multiple logistic regression analysis to determine predictor for endplate injury

\begin{tabular}{lccc} 
Parameters & Odds ratio & $95 \%$ Confidence intervals & $p$-value \\
T-score for BMD & 0.52 & $0.29-0.93$ & 0.03 \\
Cage height & 1.84 & $1.07-3.17$ & 0.03 \\
Female sex & 1.100 E8 & $0.000-0.000$ & 0.998 \\
PEEK cage & 4.571 E8 & 0.000 & 0.998 \\
\hline
\end{tabular}

BMD, bone mineral density; PEEK, polyetheetherketone. 
identify the outline of the vertebral endplate (Fig. 5). This poor fluoroscopic image might mislead surgeons during cage trial, endplate preparation, and cage insertion. Preoperative evaluation of bone quality and perioperative intensive treatment for osteoporosis could be helpful.

The second parameter correlating with endplate injury is cage height. Some authors advocate avoiding overaggressive decortication [15,16] or overdistraction [17], however, the definition of "over" remains unclear. Tohmeh et al. [4] showed that taller disc height is a risk factor in cage settling. Le et al. [1] recommended use of 8- or 10 $\mathrm{mm}$ high cage and not a cage $\geq 12 \mathrm{~mm}$ in height to achieve indirect decompression. However, they did not provide supporting data. Presently, endplate injury correlated directly with cage height, regardless of the amount of distraction gap between cage size and preoperative radiographic measurement. This means that we cannot predict the risk of the endplate injury through preoperative radiographic measurement.

As for the timing of intraoperative endplate injury, cage size trial is a more critical stage than endplate preparation or cage insertion. A cage height that cannot be pulled out easily has been recommended [14], but the appropriate size is difficult to determine only through surgeon's touch. The experience of the surgeon did not make a significant difference in terms of the incidence of endplate injury. Despite surgeon experience or taller preoperative disc height, $20 \%$ or more of the cases that applied a cage taller than $12 \mathrm{~mm}$ demonstrated an endplate injury.

The injury rate increased markedly for a cage height of $10 \mathrm{~mm}$ to $12 \mathrm{~mm}$, suggesting that surgeons should not use a cage height exceeding $11 \mathrm{~mm}$, especially for patients with reduced BMD. For thoracic spine, an even lower height cage presents a potential risk of endplate injury.

In terms of cage material, PEEK demonstrated higher rates of endplate injury. Still, it is difficult to conclude that the material correlates directly with endplate injury. Because all cages were inserted with use of two sliders, it is difficult to attribute the endplate injury to cage material. Titanium cages were used only for the initial 32 levels in this series. For those initial cases that underwent XLIF, we were still skeptical of the effect of indirect decompression and performed direct posterior decompression for almost all cases and were reluctant to insert a taller cage. This might have reduced the rate of endplate injury in the titanium cage series.

There are several study limitations. XLIF was introduced to our country only in February 2013. So, our series has a short follow-up and the impact of intraoperative endplate injury on bony fusion or clinical outcome is still unclear. Still, all cases in our series were supplemented with bilateral pedicle screws, which provide the most rigid fixation [18]. This additional fixation might minimize the clinical impact of endplate injury. Accuracy of the radiographic measurements was not completely verified, even though we performed preoperative measurements on CT-MPR instead of standing plain X-rays to achieve more accurate and reproducible measurements, especially in deformity cases and to simulate intraoperative segmental contour in lateral position. Finally, we could not analyze the effect of the cage width on endplate injury, because only the 18 $\mathrm{mm}$ cage is available in our country. This situation might account for different results from previous studies $[1,4,6]$.

\section{Conclusions}

Intraoperative endplate injury occurred in $10.4 \%$ of our initial 102 consecutive XLIF cases. The predisposing factors of endplate injury were reduced BMD and cage height regardless of the amount of distraction. Precise preoperative evaluation of bone quality, intensive perioperative treatment for osteoporotic cases and cautious choice of cage height may have reduced the incidence of endplate injury. Longer follow-up is necessary to reveal the impact of intraoperative endplate injury on clinical outcome.

\section{Conflict of Interest}

No potential conflict of interest relevant to this article was reported.

\section{References}

1. Le TV, Baaj AA, Dakwar E, et al. Subsidence of polyetheretherketone intervertebral cages in minimally invasive lateral retroperitoneal transpsoas lumbar interbody fusion. Spine (Phila Pa 1976) 2012;37:126873.

2. Marchi L, Abdala N, Oliveira L, Amaral R, Coutinho E, Pimenta L. Radiographic and clinical evaluation of cage subsidence after stand-alone lateral interbody fusion. J Neurosurg Spine 2013;19:110-8.

3. Malham GM, Ellis NJ, Parker RM, Seex KA. Clinical outcome and fusion rates after the first 30 extreme 
lateral interbody fusions. ScientificWorldJournal 2012;2012:246989.

4. Tohmeh AG, Khorsand D, Watson B, Zielinski X. Radiographical and clinical evaluation of extreme lateral interbody fusion: effects of cage size and instrumentation type with a minimum of 1-year follow-up. Spine (Phila Pa 1976) 2014;39:E1582-91.

5. Oxland TR, Grant JP, Dvorak MF, Fisher CG. Effects of endplate removal on the structural properties of the lower lumbar vertebral bodies. Spine (Phila $\mathrm{Pa}$ 1976) 2003;28:771-7.

6. Ozgur BM, Aryan HE, Pimenta L, Taylor WR. Extreme Lateral Interbody Fusion (XLIF): a novel surgical technique for anterior lumbar interbody fusion. Spine J 2006;6:435-43.

7. Belkoff SM, Maroney M, Fenton DC, Mathis JM. An in vitro biomechanical evaluation of bone cements used in percutaneous vertebroplasty. Bone 1999;25(2 Suppl):23S-26S.

8. Hou Y, Luo Z. A study on the structural properties of the lumbar endplate: histological structure, the effect of bone density, and spinal level. Spine (Phila Pa 1976) 2009;34:E427-33.

9. Park SH, Park WM, Park CW, Kang KS, Lee YK, Lim SR. Minimally invasive anterior lumbar interbody fusion followed by percutaneous translaminar facet screw fixation in elderly patients. J Neurosurg Spine 2009;10:610-6.

10. Vaidya R, Sethi A, Bartol S, Jacobson M, Coe C, Craig JG. Complications in the use of rhBMP-2 in PEEK cages for interbody spinal fusions. J Spinal
Disord Tech 2008;21:557-62.

11. Kozak JA, Heilman AE, O'Brien JP. Anterior lumbar fusion options: technique and graft materials. Clin Orthop Relat Res 1994;(300):45-51.

12. Grant JP, Oxland TR, Dvorak MF. Mapping the structural properties of the lumbosacral vertebral endplates. Spine (Phila Pa 1976) 2001;26:889-96.

13. Steffen T, Tsantrizos A, Aebi M. Effect of implant design and endplate preparation on the compressive strength of interbody fusion constructs. Spine (Phila Pa 1976) 2000;25:1077-84.

14. Rodgers WB, Uribe JS. Complications and their avoidance in XLIF ${ }^{\circ}$. In: Goodrich JA, Volcan IJ, editors. eXtreme Lateral Interbody Fusion $\left(\mathrm{XLIF}^{\oplus}\right)$. 2nd ed. St. Louis: Quality Medical Publishing Inc.; 2013. p.179-97.

15. Billinghurst J, Akbarnia BA. Extreme lateral interbody fusion-XLIF. Curr Orthop Pract 2009;20:23851.

16. Schiffman M, Brau SA, Henderson R, Gimmestad G. Bilateral implantation of low-profile interbody fusion cages: subsidence, lordosis, and fusion analysis. Spine J 2003;3:377-87.

17. Truumees E, Demetropoulos CK, Yang KH, Herkowitz HN. Effects of disc height and distractive forces on graft compression in an anterior cervical discectomy model. Spine (Phila Pa 1976) 2002;27:2441-5.

18. Tohmeh AG, Ryu SI, Turner AW. Fixation options in $\mathrm{XLIF}^{\bullet}$. In: Goodrich JA, Volcan IJ, editors. eXtreme Lateral Interbody Fusion $\left(\mathrm{XLIF}^{\circ}\right)$. 2nd ed. St. Louis: Quality Medical Publishing Inc.; 2013. p.117-35. 